M. Lutz fait la communication suivante :

\title{
Comparaison de l'azote nitrique et de l'azote total dans les plantes parasites et saprophytes;
}

\author{
par M. L. LUTZ.
}

J'ai montré, dans un précédent travail ${ }^{1}$, que les plantes parasites et saprophytes renferment toutes des nitrates dans leurs tissus. J'ai constaté, en outre, que les proportions de nitre accumulées étaient facteurs, à la fois du parasitisme plus ou moins complet du végétal, de la richesse du substratum et aussi de la présence ou de l'absence de chlorophylle. confirmant de la sorte les observations de Schimper ${ }^{2}$ sur l'action simultanée exercée par la lumière et la chlorophylle dans ces phénomènes d'accumulation.

Il pouvait être intéressant de comparer les quantités de nitrates ainsi inutilisées à l'azote total contenu dans les mêmes plantes, ces données constituant des renseignements sur l'activité de phénomènes assimilateurs dont les parasites sont le siège.

J'ai examiné, au point de vue de l'azote total, la plupart des plantes précédemment étudiées. J'ai dû, faute de matériaux en quantité suffisante, en laisser de côté un petit nombre, mais, par contre, j'ai pu me procurer plusieurs autres espèces qui ont été soumises aux mêmes essais ${ }^{3}$.

Le dosage de l'azote total a été effectué par la méthode de Dumas; celui de l'azote nitrique, comme précédemment, par celle de Schlœsing, sur des extraits de plantes repris par l'alcool faible pour éliminer les matières gommeuses.

Les résultats; rapportés à 1000 grammes de poids sec sont

1. LUTz (L.), Sur l'accumulation des nitrates dans les plantes parasites et saprophytes et sur l'insuffisance de la diphénylamine sulfurique comme réactif microchimique de ces substances. Bull. Soc. bot. de Fr., t. LV, 1908, p. 104.

2. SCHimper, Ueber Kalkoxalatbildung in den Laubblättern, 4, Bot. Zeit., t. XLVI, 1888, p. 112.

3. Je remercie très cordialement nos collègues, MM. Col et A. Jahandiez, qui ont récolté à mon intention le Lathræa Clandestina et le Cytinus kermesinus. 
relatés dans le tableau ci-après, dans lequel, pour plus de commodité, j’ai rapproché l'azote nitrique et l'azote total.

\section{Parasites absolues.}

Gui.

Cuscute (sur Ortie) (École de pharmacie de

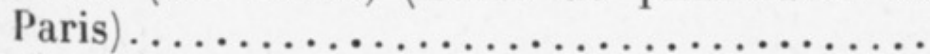

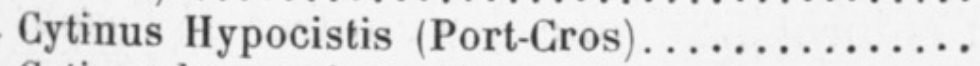

Cytinus kermesinus (Carqueiranne).........

Cynomorium coccineum, tige (dunes sableuses

près Oran).

Arceuthobium Oxycedri (env de Marseille)...

Lathræa Clandestina (env. de Nantes).......

Azote nitrique Azote total p. 1000 en gr. p. 1000 en gr.

\section{Parasites relatives à chlorophylle.}

Melampyrum arvense (Savigny-sur-Orge)......

0,589

54,83

0,263

33,53

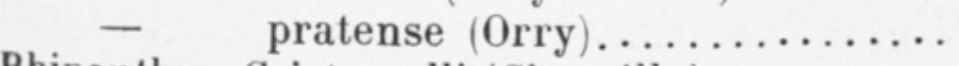

0,482

33,50

Rhinanthus Crista-galli (Chantilly).........

0,194

44,30

0,156

40,21

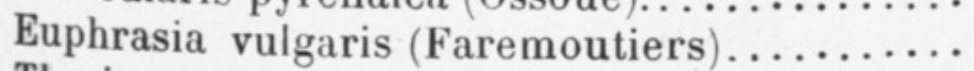

0,369

41,12

Thesium pratense (tiges) (Barcelonette)......

Osyris alba (Hyères).

0,225

38,92

- lanceolata (env. d'Oran)...........

0,0094

24,71

0,075

27,58

\section{Parasites relatives sans chlorophylle.}

Phelipæa cærulea (embouchure du Rizzanèse,

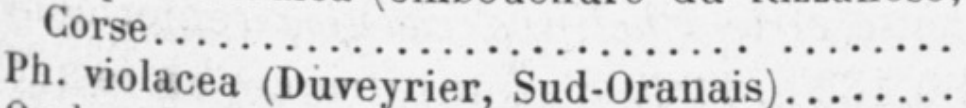

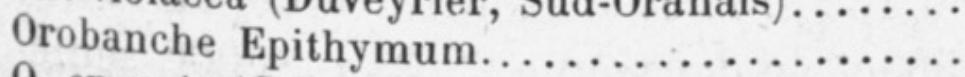

0 . cruenta (Cabourg)

0 . Rapum (Bastia).

0 . minor (Chevreuse) $\ldots \ldots \ldots \ldots \ldots \ldots \ldots \ldots$

0,301

$0,332 \quad 20,81$

\section{Saprophytes sans chlorophylle.}

$\begin{array}{llll}\text { Neottia Nidus-avis (Forêt d'Halatte) } \ldots \ldots \ldots \ldots & 0,156 & 23,75 \\ \text { Monotropa Hypopitys (Hyères) } \ldots \ldots \ldots \ldots \ldots \ldots & 0,739 & 26,04\end{array}$

Une première conclusion à tirer des chiffres de ce tableau est que les parasites absolues sont en général plus pauvres en azote total que les plantes dont une partie des racines plonge dans le sol, et y puise des sels nutritifs. Cette particularité est 
surtout marquée pour les plantes chlorophylliennes, mais les différences restent cependant moins accentuées que pour l'azote nitrique, ce qui était du reste facile à prévoir en raison du mode particulier de nutrition de ce groupe de végétaux.

Mais un fait beaucoup plus important prime celui-ci : c'est la relation qui existe entre la présence ou l'absence de chlorophylle et la richesse comparée des parasites en azote total et en azote nitrique.

Lorsque la plante est chlorophyllienne, les chiffres de l'azote nitrique sont faibles, ceux de l'azote total élevés et réciproquement.

Certains exemples sont particulièrement caractéristiques :

Le Pedicularis pyrenaica et le Neottia Nidus-avis renferment tous les deux 0,156 p. 1000 d'azote nitrique, mais le premier, plante à chlorophylle, contient 40,21 p. 1000 d'azote total et le second 23,75 p. 1000 seulement;

Le Gui et le Cytinus Hypocistis titrent tous deux 0,07็ p. 1000 d'azote nitrique, mais le premier a une teneur de 35,14 p. 1000 d'azote total et le second de 27,67;

Le Thesium pratense, l'Orobanche rapum (Bastia) et l'O. minor renferment : le premier 0,225 , le second et le troisième 0,257 p. 1000 d'azote nitrique, mais tandis que le Thesium contient 38,92 p. 1000 d'azote total, l'Orobanche Rapum n'en contient que 29,12 et l' $O$. minor 20,95 ;

Le Melampyrum pratense et le Phelipæa cærulea renferment respectivement 0,482 et 0,489 p. 1000 d'azote nitrique, alors que le premier contient 33,50 et le deuxième 22,34 p. 1000 seulement d'azote total;

Le Melampyrum arvense (Savigny) et l'Orobanche cruenta ont des teneurs en azote nitrique de 0,589 et 0,551 p. 1000 et l'azote total y atteint respectivement 54,83 et 31,26 p. 1000 ;

Enfin le Monotropa Hypopitys, qui contient la dose élevée de 0,739 p. 1000 d'azote nitrique ne possède que 26,04 p. 1000 d'azote total.

Il est inutile d'insister plus longtemps sur ces chiffres qui montrent toute l'importance de l'action chlorophyllienne dans ces phénomènes d'accumulation et d'assimilation de l'azote chez les plantes parasites et saprophytes. 
Quelques données numériques restent cependant à souligner.

La première est relative au Phelipxa violacea, chez lequel, au contraire des autres parasites sans chlorophylle la proportion de l'azote nitrique est faible $(0,188$ p. 1000), alors que celle de l'azote total est élevée $(44,32$ p. 1000).

On sait que des travaux récents tendent à reconnaître à certains pigments autres que la chlorophylle un pouvoir assimilateur, et l'on pourrait être tenté de chercher dans cette voie une explication de cette anomalie. Je me garderai cependant d'émettre ici une telle hypothèse, car elle ne reposerait que sur un seul fait, ce qui est notoirement insuffisant pour conclure. Je me contenterai d'appeler l'attention sur ce point particulier, en attendant qu'une étude plus complète des échanges nutritifs chez cette plante fasse connaître s'il y a là une simple anomalie ou un fait se rattachant à l'histoire des synthèses végétales non chlorophylliennes.

Les autres données à remarquer sont celles fournies par l'analyse des deux espèces d'Osyris, chez lesquels on observe une teneur très faible en azote nitrique et une proportion d'azote total qui, bien que faible, est plus élevée comparativement que chez les autres parasites relatives à chlorophylle. Je me borne pour le moment à enregistrer ce fait : le parasitisme des Osyris est actuellement l'objet de recherches et il est probable que, personnellement, j'aurai à revenir prochainement sur leurs échanges nutritifs.

\title{
M. F. Camus résume le travail ci-dessous :
}

\section{Plantes nouvelles, rares ou critiques}

\author{
(Suite) ';
}

PAR MM. LES AbBés COSTE et SOULiÉ.

Saponaria bellidifolia Smith, retrouvé dans les Pyrénées. Le baron Picot de Lapeyrouse est le premier botaniste qui ait signalé cette plante dans la chaîne des Pyrénées et en France. Dans son célèbre ouvrage, Histoire abrégée des Plantes des

1. Voir t. LVIII (1911), pp. 319, 412, 533 et 577. 


\section{$2 \mathrm{BHL}$ Biodiversity Heritage Library}

Lutz, M L . 1912. "Comparaison de l'azote nitrique et de l'azote total dans les plantes parasites et saprophytes." Bulletin de la Société botanique de France 59, 370-373. https://doi.org/10.1080/00378941.1912.10832434.

View This Item Online: https://www.biodiversitylibrary.org/item/8681

DOI: https://doi.org/10.1080/00378941.1912.10832434

Permalink: https://www.biodiversitylibrary.org/partpdf/157680

\section{Holding Institution}

Missouri Botanical Garden, Peter H. Raven Library

\section{Sponsored by}

Missouri Botanical Garden

\section{Copyright \& Reuse}

Copyright Status: Public domain. The BHL considers that this work is no longer under copyright protection.

This document was created from content at the Biodiversity Heritage Library, the world's largest open access digital library for biodiversity literature and archives. Visit BHL at https://www.biodiversitylibrary.org. 